\title{
PACKAGING PAPER COATED WITH PLA
}

\author{
Diana Gregor-Svetec (D), Tadeja Šumrada \\ University of Ljubljana, Faculty of Natural Sciences and Engineering, Department of Textiles, \\ Graphic Arts and Design, Chair of Information and Graphic Arts Technology, Ljubljana, Slovenia
}

Abstract: Nowadays, eco-design of products is becoming one of the most important directions at packaging development. Efficient use of materials, use of materials from renewable sources, design for recycling and reuse are especially important. In our research, we were interested in the development of sustainable, biodegradable protective paper packaging. In order to make packaging resistant against water, paper was coated with a biodegradable biopolymer, instead of using synthetic polymer. The base packaging paper was coated with a PLA solution, which was obtained by dissolving PLA pellets in dichloromethane. Two different concentrations were used. Technological and surface properties of coated paper were determined. The results of analyses have shown that sustainable packaging paper with good surface properties and barrier against water can be obtained by coating it with a PLA solution. Comparison with a commercial packaging paper has shown, that for PLA coated paper even higher water resistance was obtained.

Key words: packaging paper, PLA, coating, surface properties

\section{INTRODUCTION}

Today, sustainable development and eco-design of products are important directions in all areas. Ecodesign is an approach to designing products with special consideration for the environmental impacts of the product during its whole lifecycle and is becoming a core design concept in packaging. (Birkeland, 2002) The main purpose of packaging is to contain and protect the packed items from their point of production through to the point of use. The challenge is to do so by optimizing the use of materials, water and energy, minimizing waste and maximizing the recovery of used packaging (Incpen, 2008). The sustainable packaging design principles of effective, efficient, cyclic, and safe should be consider (Park et al, 2014). For packaging with short-life span is even more important to focus on material choice, to use materials from renewable sources, that are recyclable or compostable, and design for maximum sustainability and recoverability (Birkeland, 2002; McDonough and Braungart, 2009).

Protective paper packaging has lot of uses, from packing products for transport to protecting them in storage, from wrapping product or filling the space inside the box. Paper is widely used in packaging applications and is biodegradable and therefore environmentally friendly material. The barrier resistance and wettability of papers are commonly controlled by the application of petroleum-based derivatives such as polyethylene, waxes and/or fluor-derivatives as coating (Rastogi and Samyn, 2015). Because of such coated layer, protective paper packaging loses its biodegradation and recyclability characteristics (Khwaldia et al, 2010). Petroleum-based polymers represent the largest segment of global waste, because of their limited recyclability and non-biodegradability. As alternative, naturally renewable biopolymers can be used as barrier coatings on paper packaging materials. Polylactide (PLA) is one of the most promising polymers because of its biocompatibility, biodegradability and the fact that it can be produced from the bio based feedstocks (Vukić et al, 2018). PLA is extremely versatile and can be injection molded, extruded, foamed, thermoformed or applied onto the paper with different coating techniques (Khwaldia et al, 2010; Vartiainen et al, 2004). In our research, we used PLA solution for coating. We prepared PLA solutions in different concentrations and used them for coating the base paper sheets, analyzed them and compared with a commercial packaging paper.

\section{METHODS}

\subsection{Materials}

One side coated, woodfree flexible packaging paper with high surface quality was used as a reference paper. For our research, a base uncoated paper of the mentioned commercial packaging paper was supplied by the producer. 
PLA pellets (6201D, Natureworks, USA) were dissolved in the solvent dichloromethane (DCM) at raised temperature. In this way, two coating solutions were prepared, in concentrations of 15 wt $\%$ and 20 wt $\%$ (dry solids weight). PLA pellets were dried in a vacuum oven at $60^{\circ} \mathrm{C}$ for $24 \mathrm{~h}$ before use. Analytical grade dichloromethane (DCM) was obtained from Carl Roth, Germany.

Coating was carried out at ambient temperature on a lab coater (RK Print-Coat Instruments, Hertz, UK) using a wire-wound bar. The coat weight was varied by varying the diameter of the wire on the bar. Two bars were used, No. 3 with the wire diameter of 0,31 mm and No. 6 with the wire diameter of 0,76 mm. After coating, the samples were allowed to dry overnight at the room temperature.

Sample identification and description is summarised in Table 1.

Table 1: Sample identification and description

\begin{tabular}{|l|l|}
\hline Sample identification & Sample description \\
\hline BP & Base paper, uncoated packaging paper \\
\hline BP-15-3 & Base paper; coated with PLA solution with concentration of 15 wt\%; bar No. 3 \\
\hline BP-15-6 & Base paper; coated with PLA solution with concentration of $15 \mathrm{wt} \%$; bar No. 6 \\
\hline BP-20-3 & Base paper; coated with PLA solution with concentration of $20 \mathrm{wt} \%$; bar No. 3 \\
\hline BP-20-6 & Base paper; coated with PLA solution with concentration of $20 \mathrm{wt} \% ;$ bar No. 6 \\
\hline CP & Commercial product; one-side coated packaging paper \\
\hline
\end{tabular}

\subsection{Methods}

Before testing, all samples were conditioned at $23^{\circ} \mathrm{C}$ and $50 \% \mathrm{RH}$. Basis weight (grammage) was determined with an analytical balance Mettler Type AE200 (Ohio, USA) with readability of 0.1 mg. Coating weight was obtained by subtracting from the weight of a defined area of coated paper, the weight of the same size area of the uncoated paper. Thickness of samples was measured in accordance with ISO 534, using micrometer Mitutoyo (Kawasaki, Japan) with $0.001 \mathrm{~mm}$ accuracy.

Surface properties were tested according to their respective standards. Roughness was measured using Bendtsen N3500 roughness tester (PTA Group, France) in accordance with ISO 8791-2. With the same measuring device the air permeance was determined following ISO 5636-3. Spectrophotometer X-Rite i1Pro2 (Michingan, USA) was used to measure ISO brightness (ISO 2470-1), opacity (ISO 2471) and colour (ISO 5631-1). Water absorbency was tested with Cobb method according to ISO 535, Klemm method according to ISO 8787 and the water absorbency rate according to Tappi T432. The morphology of the samples was examined with the Scanning electron microscopy (SEM). Prior to analysis, the samples were coated with a thin layer of gold. The images were captured with a SEM system (JSM-6060 LV, Jeol, Japan) equipped with a detector of secondary electrons.

\section{RESULTS AND DISCUSSION}

A single-layer method was used to apply PLA solution onto the base woodfree packaging paper. Two concentrations of PLA solution, 15 and $20 \mathrm{wt} \%$, were used. To deposit the coating solution on the paper, a wire-wound bar was used. Using standard wire-wound bar an uniform and repeatable coating was obtained. Two bars with different wire diameter were used, No. 3 and No. 6, for depositing 24 and $60 \mu \mathrm{m}$ wet film onto the substrate, which resulted in four different coat weights: 6 and $9 \mathrm{~g} / \mathrm{m}^{2}$ at lower PLA solution concentration, 8 and $11 \mathrm{~g} / \mathrm{m}^{2}$ at higher concentration.
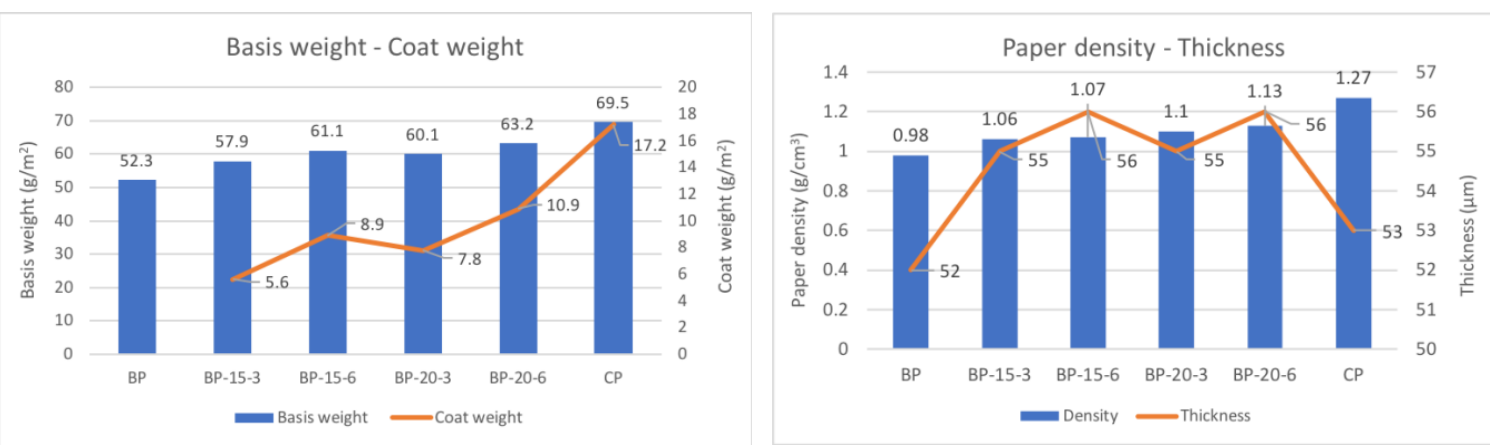

Figure 1: Technological properties of paper samples 

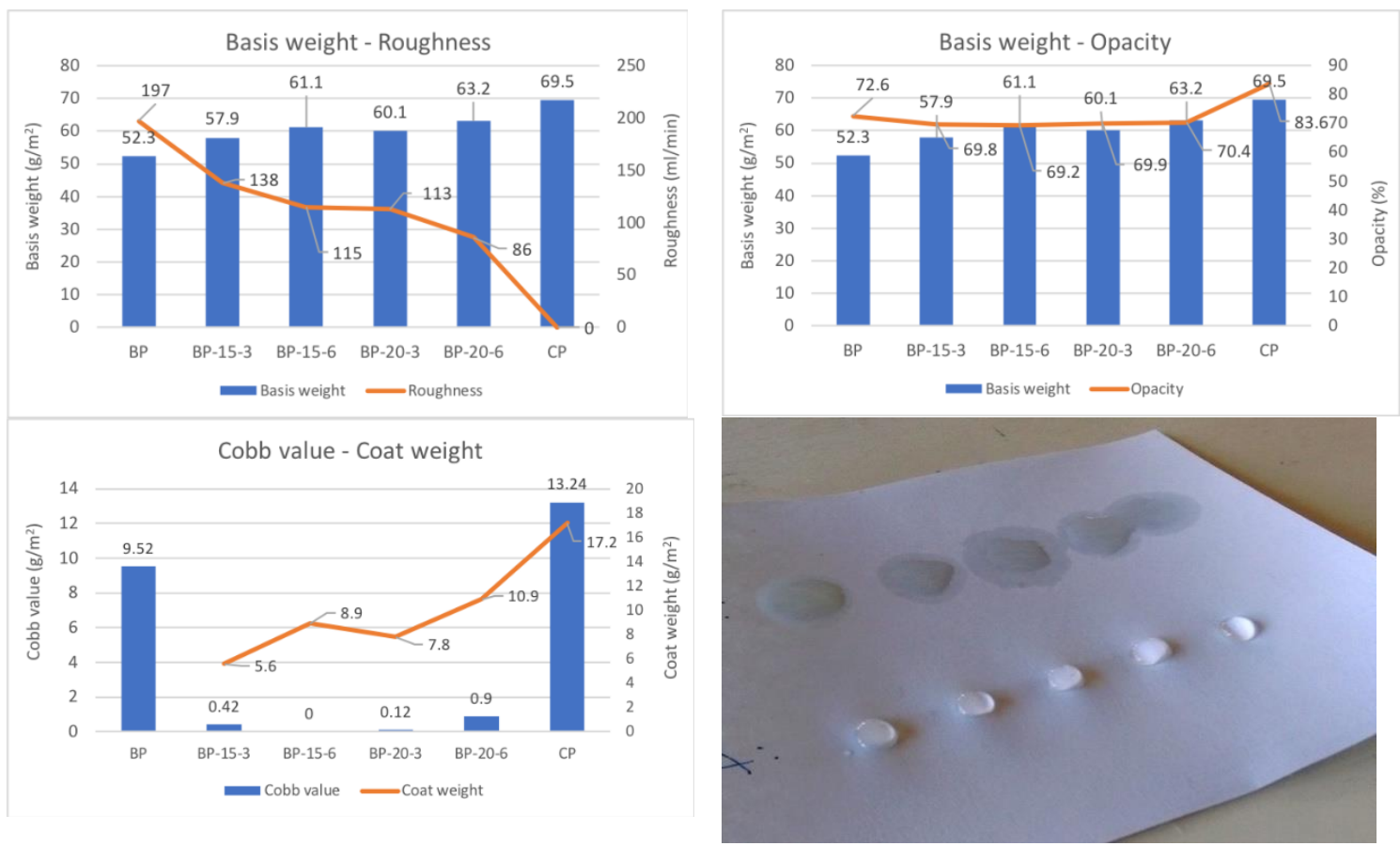

Figure 2: Surface properties of paper samples

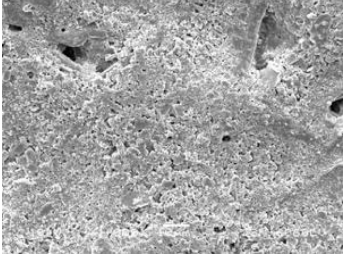

BP - top side

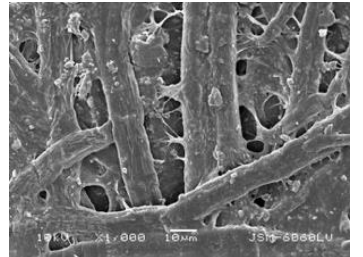

$\mathrm{BP}$ - reverse side

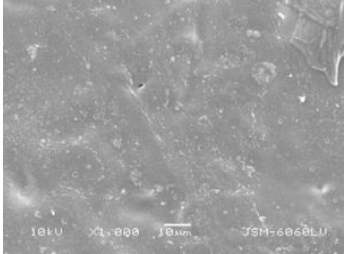

BP-15-3 - top side

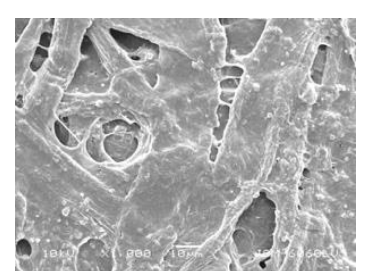

BP-15-3 - reverse side

Figure 3: SEM images of paper surface

Table 2: Colorimetric properties of paper samples

\begin{tabular}{|l|c|c|c|c|}
\hline Sample & $\mathrm{L}^{*}(/)$ & $\mathrm{a}^{*}(/)$ & $\mathrm{b}^{*}(/)$ & ISO brightness (\%) \\
\hline BP & 96,07 & 0,635 & 0,938 & 83,9 \\
\hline BP-15-3 & 95,65 & 0,656 & 1,102 & 82,4 \\
\hline BP-15-6 & 95,41 & 0,655 & 1,045 & 82,1 \\
\hline BP-20-3 & 95,75 & 0,654 & 1,021 & 83,2 \\
\hline BP-20-6 & 95,35 & 0,637 & 0,718 & 82,6 \\
\hline CP & 93,5 & 1,179 & $-1,226$ & 81,1 \\
\hline
\end{tabular}

Coat weight is in very high correlation with the basis weight, thickness and paper density, as expected (Figure 1). It is obvious, that thickness depends more on the volume of the deposited PLA solution onto the paper and less on the solution concentration (Figure 4). Base paper was precoated on one side, as seen from the Figure 3. Surface sizing has improved barrier and surface properties of base paper and gave good foundation for coating. The smoothness of paper surface was raised with coating, but mainly barrier properties improved, especially barrier properties to water (Figure 2). With increasing coat weight, the surface roughness decreased (Figure 4).

Coating with the PLA solution closed the surface, which resulted in very low water absorptiveness into the surface (Cobb value around $0 \mathrm{~g} / \mathrm{m}^{2}$, water droplet didn't change its shape in 10 minutes) and no water absorbency due to capillary action (Klemm value bellow $1 \mathrm{~mm}$ ). Already, lower concentration of PLA solution (15 wt\%) and lower volume of wet film deposited (bar No. 3) resulted in excellent paper barrier properties to water. As seen from the Figure 3, the top side of the paper is completely covered with the PLA polymer, and even reverse side of the paper is partly coated. PLA solution obviously penetrated into the paper and closed also the pores in the paper, not only voids on the surface of the paper. This was 
confirmed by the measurement of air permenance, which showed that no air has penetrated through the paper. That is way increasing the concentration of PLA solution or volume of deposited solution on the paper had little effect on improvement of surface properties (Figure 4).

To check if coating suspension has any influence on color of the paper surface, the CIELab values and ISO brightness were determined. With coating ISO brightness decreases, though the difference is small, below 2\% (Table 2). Lower values were obtained at lower concentration of PLA solution and higher volume of deposited solution on the paper (Figure 4). Also, a small decrease in opacity is seen. The color differences between base paper and coated samples are negligible, $\Delta \mathrm{E}$ is bellow 1 (Table 2).

Compared to reference sample, commercially produced one-side coated packaging paper, PLA coated papers have lower basis weight and density, and higher thickness. Commercially produced coated paper is calendred, which increases density and lowers the thickness of paper. The coat weight at commercial product is higher compared to PLA coated papers, which has some influence on surface properties, though lower than expected. A bit higher opacity and smoothness, lower ISO brightness, and higher Cobb value are more connected to the composition of coated layer and finishing process and less with the coat weight.
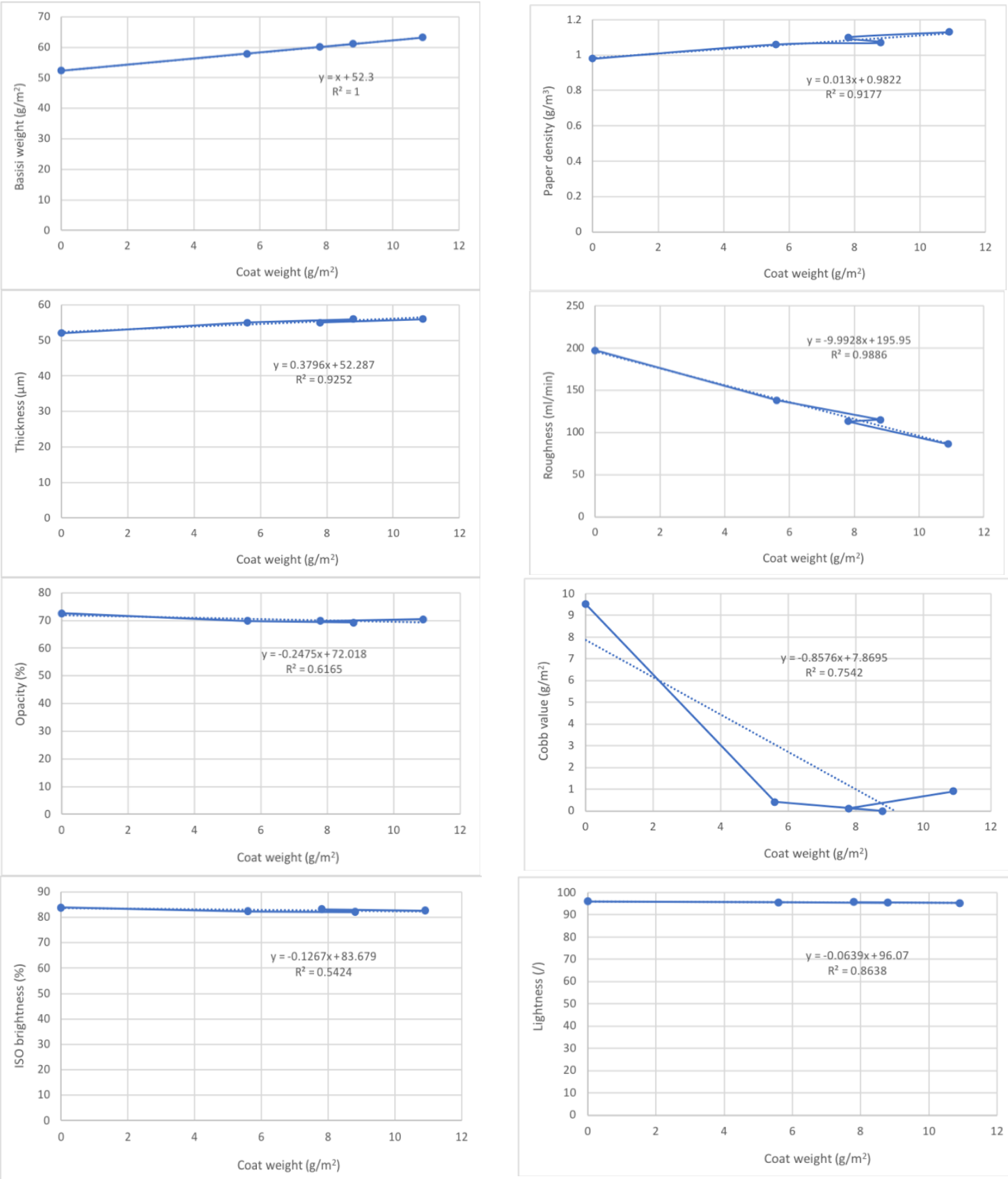

Figure 4: Dependence between coat weight and paper properties 


\section{CONCLUSIONS}

In the present research, packaging paper was made by coating the base paper with the PLA solution, with two different concentrations. The comparison with the commercial packaging paper has shown that similar surface properties were obtained. The influence of coating on optical properties of paper is small, roughness and brightness decrease a little, opacity is higher, colour change is negligible. The analysis has shown, that already lower concentrations of PLA solution enable uniform coating, which lowers permeability and gives good barrier properties to water. Higher concentrations of PLA solution further improve barrier properties, but also has a drawback. Because in this case, the non-uniformity of coated surface also increases, the variation in paper properties is higher. The results of analysis have shown that eco-friendly packaging paper with good resistance to water can be obtained by coating with a suitable concentration of PLA solution.

\section{ACKNOWLEDGMENTS}

This work was supported by the Slovenian Research Agency (Programme P2-0213).

\section{REFERENCES}

[1] Birkeland, J.: "Design for sustainability", (Earthscan, London, 2002.)

[2] Envirowise, Packguide: a guide to packaging eco-design, Incpen. URL: http://www.packagingfedn.co.uk/images/reports/Incpen\&Envirowise\%20Guide\%20to\%20Packaging \%20Eco\%20Design.pdf (last request: 2020-06-21.).

[3] Khwaldia K., Arab-Tehrany E., Desobry, S.: "Biopolymer Coatings on Paper Packaging Materials", Comprehensive reviews in food science and food safety 9 (3), 82-91, 2010. doi: 10.1111/j.15414337.2009.00095.x.

[4] McDonough, W., Braungart, M.: "Cradle to cradle: remaking the way we make things", (Vintage, London, 2009.).

[5] Park, S-I., Lee, D.S., Han, J.H.: "Innovations in Food Packaging", (Academic Press, 2014.), pages 537-547.

[6] Rastogi, V.K., Samyn, P.: "Bio-Based Coatings for Paper Applications", Coatings 5 (4), 887-930, 2015. doi: 10.3390/coatings5040887.

[7] Vartiainen, J., Motion, R., Kulonen, H., Ratto, M., Skytta, E., Ahvenainen, R.: "Chitosan-coated paper: effects of nisin and different acids on the antimicrobial activity", Journal of Applied Polymer Science 94, 986-93, 2004. doi: 10.1002/app.20701.

[8] Vukić, N., Erceg, T., Teofilović, V., Nikolić, L., Cakić, S., Simendić, B., Ristić, I.: "The use of the green chemistry concept in the synthesis of packaging material based on polylactide", Proceedings of GRID 2018, (Faculty of Technical Sciences: Novi Sad, Serbia, 2018), pages 281-287.

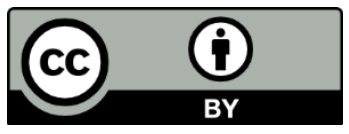

(C) 2020 Authors. Published by the University of Novi Sad, Faculty of Technical Sciences, Department of Graphic Engineering and Design. This article is an open access article distributed under the terms and conditions of the Creative Commons Attribution license 3.0 Serbia (http://creativecommons.org/licenses/by/3.0/rs/). 